\title{
A global solution curve for a class of free boundary value problems arising in plasma physics
}

\author{
Philip Korman \\ Department of Mathematical Sciences \\ University of Cincinnati \\ Cincinnati Ohio 45221-0025
}

\begin{abstract}
We study the existence and multiplicity of solutions and the global solution curve of the following free boundary value problem, arising in plasma physics, see H. Berestycki and H. Brezis [3] find a function $u(x)$ and a constant $b$, satisfying

$$
\Delta u+g(x, u)=p(x) \text { in } D
$$$$
\left.u\right|_{\partial D}=b, \quad \int_{\partial D} \frac{\partial u}{\partial n} d s=0 .
$$

Here $D \subset R^{n}$, is a bounded domain, with smooth boundary. This problem can be seen as a PDE generalization of the periodic problem for one-dimensional pendulum-like equations. We use continuation techniques. Our approach is suitable for numerical computations.
\end{abstract}

Key words: Free boundary value problem, global solution curve.

AMS subject classification: 35J15, 78A30.

\section{Introduction}

We study the existence and multiplicity of solutions, and the global solution structure of the following free boundary value problem, arising in plasma physics, see H. Berestycki and H. Brezis [3]. On a bounded domain $D \subset R^{n}$, with a smooth boundary, we seek to find a function $u(x)$ and a constant $b$, satisfying

$$
\begin{gathered}
\Delta u+g(x, u)=p(x) \text { in } D \\
\left.u\right|_{\partial D}=b, \quad \int_{\partial D} \frac{\partial u}{\partial n} d s=0 .
\end{gathered}
$$


Here $g(x, u)$ and $p(x)$ are given functions, $n$ denotes the unit normal vector on $\partial D$, pointing outside. More recently, this problem was studied in P. Amster, P.L. De Napoli, and M.C. Mariani [2]. As explained in [2], this problem can be seen as a PDE generalization of the periodic problem for the one-dimensional pendulum-like equation:

$$
\begin{gathered}
u^{\prime \prime}+g(t, u)=p(t) \\
u(0)=u(T)=b, \quad u^{\prime}(0)=u^{\prime}(T) .
\end{gathered}
$$

with $p(t)$ being $T$-periodic, and $g(t, u)$ is $T$-periodic in $t$. Indeed, writing the second boundary condition in (1.2) as $\int_{0}^{T} u^{\prime \prime} d t=0$, we see that a similar condition for (1.1) is $\int_{D} \Delta u d x=0$, which by the divergence theorem is equivalent to the second boundary condition in (1.1). There exists an enormous literature for the periodic problem (1.2), see e.g., the review paper of J. Mawhin [13, and [5], 7], 8], [14], [15], [16]. By constrast, not much is known for the more general problem (1.1).

We use continuation techniques to study the solution curves for the problem (1.1), similarly to our approach to the pendulum-like equations [9]. One can think of the problem (1.1) as being "at resonance", i.e., some conditions on $p(x)$ are necessary for its solvability. Indeed, decompose $p(x)=\mu_{0}+\theta(x)$, with $\mu_{0}=\int_{D} p(x) d x$, and $\int_{D} \theta(x) d x=0$. Integrating the equation in (1.1), we get

$$
\int_{D} g(x, u(x)) d x=\mu_{0}|D|
$$

where $|D|$ is the volume of $D$. Assume that the following limits exist: $g(x, \pm \infty)=\lim _{u \rightarrow \pm \infty} g(x, u)$, uniformly in $x \in \bar{D}$, with $g(x, \pm \infty) \in L^{\infty}(D)$, and suppose that

$$
g(x,-\infty)<g(x, u)<g(x, \infty) \text { for all } x \in D .
$$

Then, from (1.3) and (1.4), we obtain

$$
\frac{1}{|D|} \int_{D} g(x,-\infty) d x<\mu_{0}<\frac{1}{|D|} \int_{D} g(x, \infty) d x
$$

to be a necessary condition for existence of solutions. Similarly to the classical paper of E.M. Landesman and A.C. Lazer [12], we give conditions under which the condition (1.5) is sufficient for existence.

In order to use continuation techniques, we will embed the problem (1.1) into a family of problems

$$
\begin{aligned}
& \Delta u+k g(x, u)=p(x) \text { in } D \\
& \left.u\right|_{\partial D}=b, \quad \int_{\partial D} \frac{\partial u}{\partial n} d s=0,
\end{aligned}
$$


with $0 \leq k \leq 1$. At $k=1$, one recovers the original problem (1.1), while at $k=0$ the equation is linear. It turns out that at $k=0$, the problem has infinitely many solutions, and one may fix a unique solution, by additionally

prescribing any value of $\frac{1}{|D|} \int_{D} u(x) d x \equiv \xi_{1}$. One then performs continuation in $k$ on curves of fixed average. Namely, for any $\xi_{1} \in R$, one solves the following problem: find $(u, b, \mu)$, as a function of $k$, solving

$$
\begin{gathered}
\Delta u+k g(x, u)=\mu+\theta(x) \text { in } D \\
\left.u\right|_{\partial D}=b, \quad \int_{\partial D} \frac{\partial u}{\partial n} d s=0 \\
\frac{1}{|D|} \int_{D} u(x) d x=\xi_{1} .
\end{gathered}
$$

To prove the solvability of (1.7), we set up the appropriate function spaces, and show that the corresponding differential operator is one-to-one, and onto, so that the Implicit Function Theorem applies. "Onto" is the harder part. Once the continuation process is completed, and one has a solution of the problem (1.7) at $k=1$, it remains to show that one can select a value of $\xi_{1}$, at which $\mu=\mu_{0}$, thus giving us a solution of the original problem (1.1). This part is also accomplished by continuation.

In addition to a result of Landesman-Lazer type, we obtain an existence result of D.G. de Figueiredo and W.-M. Ni [6] type, which does not require that limits at infinity exist. We show that $\xi_{1}$ is a global parameter, and then we study the curve $\mu=\mu\left(\xi_{1}\right)$, yielding a multiplicity result. The continuation approach of this paper is similar to our paper on pendulumlike equations, see [9]. In addition to its conceptual clarity, this approach is suitable for numerical computation of all solutions of (1.1). Every solution can be obtained by two continuations, first in $k, 0 \leq k \leq 1$, and then in $\xi_{1}$. These solutions curves are easy to compute numerically e.g., by the predictor-corrector method, since we prove that no turning points (or other singularities) are encountered. We had performed similar numerical computations in [10].

\section{Continuation of solutions of any fixed average}

We begin with the following linear problem

$$
\begin{gathered}
\Delta u=\theta(x) \text { in } D \\
\left.u\right|_{\partial D}=b, \quad \int_{\partial D} \frac{\partial u}{\partial n} d s=0 \\
\frac{1}{|D|} \int_{D} u(x) d x=\xi_{1} .
\end{gathered}
$$


Lemma 2.1 Given any $\theta(x) \in L^{2}(D)$, with $\int_{D} \theta(x) d x=0$, and any $\xi_{1} \in R$, we can find a unique pair $(u, b) \in W^{2,2}(D) \cap W_{0}^{1,2}(D) \times R$, solving (2.1).

Proof: $\quad$ Let $v(x) \in W^{2,2}(D) \cap W_{0}^{1,2}(D)$ be the solution of

$$
\Delta v=\theta(x) \text { in } D, v=0 \text { on } \partial D .
$$

By the divergence theorem, $\int_{\partial D} \frac{\partial v}{\partial n} d s=0$. Then $u=v+b$ will give us solution of (2.1), if we select the constant $b$, so that $\frac{1}{D} \int_{D}(v+b) d x=\xi_{1}$. Uniqueness follows from the fact that any harmonic in $D$ function, satisfying $\int_{\partial D} \frac{\partial u}{\partial n} d s=0$, is a constant.

Corollary 1 Assume in addition that $\theta(x) \in L^{\infty}(D)$, and $\xi_{1}=0$. Then there is a constant $c$, such that

$$
\|u\|_{L^{\infty}(\bar{D})} \leq c
$$

Proof: Using the $W^{2, p}$ estimates and the Sobolev embedding theorem, we conclude an estimate for $\|v\|_{L^{\infty}(\bar{D})}$, and then for $|b|$, giving us an estimate of $u=v+b$.

The following Poincare's inequality is well known. By $c_{0}$ we denote the best (largest) constant.

Lemma 2.2 Assume that $u(x) \in W_{0}^{1,2}(D)$ is any function satisfying $\int_{D} u(x) d x=$ 0 . Then there is a constant $c_{0}$, depending only on $D$, such that

$$
\int_{D}|\nabla u|^{2} d x \geq c_{0} \int_{D} u^{2} d x
$$

We consider next the following linear problem: given the function $a(x)$, find a triple $\left(w(x), \mu^{*}, b\right) \in W^{2,2}(D) \times R \times R$, satisfying

$$
\begin{gathered}
\Delta w+a(x) w=\mu^{*}, \quad \text { in } D \\
\left.w\right|_{\partial D}=b, \quad \int_{\partial D} \frac{\partial w}{\partial n} d s=0 \\
\int_{D} w(x) d x=0 .
\end{gathered}
$$

Lemma 2.3 Assume that

$$
a(x)<c_{0}, \text { for all } x \in D .
$$

Then the only solution of the problem (2.2) is $w(x) \equiv 0, \mu^{*}=0, b=0$. 
Proof: Multiply the equation in (2.2) by $w$, and integrate over $D$. Since

$$
-\int_{D} w \Delta w d x=\int_{D}|\nabla w|^{2} d x+b \int_{\partial D} \frac{\partial w}{\partial n} d s=\int_{D}|\nabla w|^{2} d x
$$

we have by Poincare's inequality

$$
c_{0} \int_{D} w^{2} d x>\int_{D} a(x) w d x=-\int_{D} w \Delta w d x=\int_{D}|\nabla w|^{2} d x \geq c_{0} \int_{D} w^{2} d x,
$$

from which we conclude that $w(x) \equiv 0$, and then $\mu^{*}=0$, and $b=0$.

Theorem 2.1 Consider the problem

$$
\begin{gathered}
\Delta u+k g(x, u)=\mu+\theta(x) \text { in } D \\
\left.u\right|_{\partial D}=b, \quad \int_{\partial D} \frac{\partial u}{\partial n} d s=0 \\
\frac{1}{|D|} \int_{D} u(x) d x=\xi_{1},
\end{gathered}
$$

where $\theta(x) \in L^{2}(D)$ is a given function. The function $u(x)$ and the constants $\mu$ and $b$ are unknown. Assume that $g(x, u) \in C^{1}(\bar{D} \times R)$ satisfies

$$
\left|g_{u}(x, u)\right| \leq M, \text { for all } x \in \bar{D} \text {, and } u \in R,
$$

and

$$
M<\min \left(c_{0}, \lambda_{2}\right) .
$$

Then for any $0 \leq k \leq 1$, and $\xi_{1} \in R$, there exists a unique solution $(u(x), b, \mu) \in W^{2,2}(D) \times R \times R$, solving (2.3).

Proof: We perform continuation in $k$. When $k=0$, we take $\mu=0$, and obtain the unique solution $(u(x), 0, b)$ by Lemma 2.1. We consider two cases.

Case I: $\xi_{1}=0$.

We shall recast the problem (2.3) in the operator form, after we define the appropriate spaces. We shall denote by $W^{2,2}(D) \cap W_{0}^{1,2}(D)+R$ the functions of the form $v(x)+b$, with $v(x) \in W^{2,2}(D) \cap W_{0}^{1,2}(D)$, and $b \in R$. Define

$\bar{H}^{2}=\left\{u(x) \in W^{2,2}(D) \cap W_{0}^{1,2}(D)+R \mid \int_{D} u(x) d x=0, \int_{\partial D} \frac{\partial u}{\partial n} d s=0\right\}$.

One checks that $\bar{H}^{2}$ is a Banach space. Consider the map

$$
F(u, \mu, k)=\Delta u+k g(x, u)-\mu: \bar{H}^{2} \times R \times R \rightarrow L^{2}(D) .
$$


Then the problem (2.3) can be written in the operator form

$$
F(u, \mu, k)=\theta(x) \text {. }
$$

Observe that the unknown constant $b$ is now "placed" in the definition of the space $\bar{H}^{2}$. We wish to apply the Implicit Function Theorem to perform continuation in $k$. For that we need to show that the linearized operator

$$
F_{(u, \mu)}(u, \mu, k)\left(w, \mu^{*}\right)=\Delta w+k g_{u}(x, u) w-\mu^{*}
$$

is one-to-one and onto.

It is one-to-one, because the only solution of the problem

$$
\begin{gathered}
\Delta w+k g_{u}(x, u) w-\mu^{*}=0, \text { in } D \\
\left.w\right|_{\partial D}=b, \quad \int_{\partial D} \frac{\partial w}{\partial n} d s=0 \\
\int_{D} w(x) d x=0
\end{gathered}
$$

is $w(x) \equiv 0, \mu^{*}=0$, in view of Lemma 2.3

Turning to the "onto" part, we need to show that given any $\theta(x) \in$ $L^{2}(D)$, one can find $w \in \bar{H}^{2}$, and $\mu^{*} \in R$, solving

$$
\begin{gathered}
\Delta w+k g_{u}(x, u) w=\mu^{*}+\theta(x), \text { in } D \\
\left.w\right|_{\partial D}=b, \quad \int_{\partial D} \frac{\partial w}{\partial n} d s=0 \\
\int_{D} w(x) d x=0 .
\end{gathered}
$$

We consider two further cases.

Case 1. The operator $L: W^{2,2}(D) \cap W_{0}^{1,2}(D) \rightarrow L^{2}(D)$, defined by

$$
L w \equiv \Delta w+k g_{u}(x, u) w, \text { subject to } w=0 \text { on } \partial D
$$

is invertible. Let $w_{1}=L^{-1}(1)$, i.e., $w_{1}$ satisfies

$$
\Delta w_{1}+k g_{u}(x, u) w_{1}=1, \text { in } D, w_{1}=0 \quad \text { on } \partial D .
$$

We also consider $z=1-L^{-1}\left(k g_{u}(x, u)\right)$, i.e., $z$ satisfies

$$
\Delta z+k g_{u}(x, u) z=0, \text { in } D, z=1 \text { on } \partial D .
$$

We shall build the solution of (2.8), by using $w_{1}$ and $z$. Multiplying (2.9) by $z$, subtracting the equation (2.10) multiplied by $w_{1}$, and integrating

$$
\int_{D} z d x=\int_{\partial D} \frac{\partial w_{1}}{\partial n} d s
$$


Sub-case i. The integrals in (2.11) are both non-zero. We now construct the solution of (2.8) in the form

$$
w=\mu^{*}\left(w_{1}+b z\right)+L^{-1}(\theta(x))+b_{1} z,
$$

with the constants $\mu^{*}, b$ and $b_{1}$ to be selected. Clearly, $w$ satisfies the equation in (2.8), while $\left.w\right|_{\partial D}=b+b_{1}$. Since $\int_{D} z d x \neq 0$, we can select $b$ and $b_{1}$, such that

$$
\int_{D}\left(w_{1}+b z\right) d x=\int_{D}\left(L^{-1}(\theta)+b_{1} z\right) d x=0 .
$$

It follows that $\int_{D} w(x) d x=0$, for any $\mu^{*}$. The function $W \equiv w_{1}+b z$ satisfies

$$
\begin{gathered}
\Delta W+k g_{u}(x, u) W=1, \text { in } D \\
\left.W\right|_{\partial D}=b, \\
\int_{D} W(x) d x=0 .
\end{gathered}
$$

By Lemma 2.3, it follows that $\int_{\partial D} \frac{\partial W}{\partial n} d s \neq 0$. Hence, we can select $\mu^{*}$, so that

$$
\int_{\partial D} \frac{\partial w}{\partial n} d s=0
$$

Sub-case ii. The integrals in (2.11) are both zero. Since $\int_{D} z(x) d x=0$, it follows by Lemma 2.3 that

$$
\int_{\partial D} \frac{\partial z}{\partial n} d s \neq 0
$$

Similarly, since $\int_{\partial D} \frac{\partial w_{1}}{\partial n} d s=0$, we conclude by Lemma 2.3 that

$$
\int_{D} w_{1}(x) d x \neq 0
$$

We now construct the solution of (2.8) in the form

$$
w=\mu^{*} w_{1}+L^{-1}(\theta(x))+b z .
$$

Clearly, $w$ satisfies the equation in (2.8), while $\left.w\right|_{\partial D}=b$. By (2.13) we can choose $\mu^{*}$, so that $\int_{D} w(x) d x=0$, for all $b$, and then we choose $b$, so that $\int_{\partial D} \frac{\partial w}{\partial n} d s=0$, by using (2.12).

Case 2. The operator $L$ is not invertible. 
Since we have assumed $k g_{u}(x, u)<\lambda_{2}$, it follows that the null space of $L$ is one dimensional, spanned by some $\bar{w}$, with $\bar{w}(x)>0$ on $D$. By the Fredholm alternative, given any $f(x) \in L^{2}(D), L^{-1}(f(x))$ is defined, i.e., the problem

$$
\Delta w+k g_{u}(x, u) w=f(x), \text { in } D, \quad w=0 \text { on } \partial D
$$

is solvable, if and only if $\int_{D} f(x) \bar{w}(x) d x=0$. We can choose a constant $s$, so that $\int_{D}(s+\theta(x)) \bar{w}(x) d x=0$, which implies that $L^{-1}(s+\theta(x))$ is defined. Similarly, we select a constant $t$, so that $L^{-1}\left(-k g_{u}(x, u)+t\right)$ is defined, and we set $z=1+L^{-1}\left(-k g_{u}(x, u)+t\right)$, i.e., $z$ satisfies

$$
\Delta z+k g_{u}(x, u) z=t, \text { in } D, z=1 \text { on } \partial D .
$$

Sub-case i. $\int_{D} z d x \neq 0$. We construct the solution of (2.8) in the form

$$
w=L^{-1}(s+\theta(x))+b_{1} z+a\left(\bar{w}+b_{2} z\right),
$$

with $s$ fixed above, and the constants $a, b_{1}$ and $b_{2}$ to be selected. We choose $b_{1}$ and $b_{2}$, so that

$$
\int_{D}\left[L^{-1}(s+\theta(x))+b_{1} z\right] d x=\int_{D}\left(\bar{w}+b_{2} z\right) d x=0 .
$$

Then $\int_{D} w(x) d x=0$, for any constant $a$. The function $W(x)=\bar{w}+b_{2} z$ satisfies

$$
\begin{gathered}
\Delta W+k g_{u}(x, u) W=b_{2} t, \text { in } D \\
\left.W\right|_{\partial D}=b_{2}, \\
\int_{D} W(x) d x=0 .
\end{gathered}
$$

By Lemma 2.3, it follows that $\int_{\partial D} \frac{\partial W}{\partial n} d s \neq 0$. We select $a$ so that $\int_{\partial D} \frac{\partial w}{\partial n} d s=$ 0 . Then $w$ satisfies (2.8). (Observe that $L w=s+\left(b_{1}+a b_{2}\right) t+\theta(x) \equiv$ $\mu^{*}+\theta(x),\left.w\right|_{\partial D}=b_{1}+a b_{2} \equiv b$.)

Sub-case ii. $\int_{D} z d x=0$. By Lemma 2.3, and (2.14), it follows that $\int_{\partial D} \frac{\partial z}{\partial n} d s \neq 0$. Let

$$
w(x)=L^{-1}(s+\theta(x))+a \bar{w}+b z .
$$

Choose the constant $a$, so that

$$
\int_{D}\left[L^{-1}(s+\theta(x))+a \bar{w}\right] d x=0 .
$$


Then, $\int_{D} w(x) d x=0$, for any $b$. By Lemma 2.3. $\int_{\partial D} \frac{\partial z}{\partial n} d s \neq 0$. Then choosing $b$, so that $\int_{\partial D} \frac{\partial w}{\partial n} d s=0$, we conclude that $w(x)$ satisfies (2.8). $\left(\right.$ Here $L w=s+b t+\theta(x) \equiv \mu^{*}+\theta(x),\left.w\right|_{\partial D}=b$.)

Case II: $\xi_{1} \neq 0$. We reduce it to the case $\xi_{1}=0$, by letting $v=u-\xi_{1}$. Then $v(x)$ satisfies

$$
\begin{gathered}
\Delta v+k g\left(x, v+\xi_{1}\right)=\mu+\theta(x) \text { in } D \\
\left.v\right|_{\partial D}=b-\xi_{1}, \quad \int_{\partial D} \frac{\partial v}{\partial n} d s=0 \\
\frac{1}{|D|} \int_{D} v(x) d x=0 .
\end{gathered}
$$

We perform the continuation in $k$ exactly the same way as before, since the bound on $\left|g_{v}\left(x, v+\xi_{1}\right)\right|$ remains the same.

We conclude that each solution of (2.3) can be continued locally in $k$. To show that solutions can be continued for all $0 \leq k \leq 1$, we need an a priori bound on $(u, \mu)(k)$. Write $u(x)=\xi_{1}+U(x)$, with $\int_{D} U(x) d x=0$. We have

$$
\begin{aligned}
& \Delta U+k g\left(x, \xi_{1}+U\right)=p(x) \text { in } D \\
& \left.U\right|_{\partial D}=b-\xi_{1}, \quad \int_{\partial D} \frac{\partial U}{\partial n} d s=0 .
\end{aligned}
$$

By our conditions (2.4) and (2.5), we can find positive constants $c_{1}$ and $c_{2}$, with $c_{1}<c_{0}$, such that

$$
\left|g\left(x, \xi_{1}+U\right)\right| \leq c_{1} U+c_{2} \text {, for all } x \in \bar{D} \text {, and } U \in R .
$$

Multiplying (2.16) by $U$, and using the Lemma 2.2, we conclude a bound on $\|U\|_{L^{2}(D)}$. Using this bound in (2.16), and the estimate (2.17), we obtain a bound on $\|U\|_{W^{2,2}(D)}$ (keep in mind that $\xi_{1}$ is arbitrary, but fixed). Writing $u=b+V$, and proceeding similarly, we conclude an $L^{2}$ bound on $V$, which implies a bound on $b$, since $\|u\|_{L^{2}(D)}$ is bounded.

Hence for any $\xi_{1}$ we have a curve of solutions $(u, \mu)(k) \in \bar{H}^{2} \times R$ solving (2.3), and at $k=1$, we have a solution of

$$
\begin{gathered}
\Delta u+g(x, u)=\mu+\theta(x) \text { in } D \\
\left.u\right|_{\partial D}=b, \quad \int_{\partial D} \frac{\partial u}{\partial n} d s=0 \\
\frac{1}{|D|} \int_{D} u(x) d x=\xi_{1} .
\end{gathered}
$$

Recall that the original problem (1.1), after we decomposed $p(x)=\mu_{0}+\theta(x)$, is

$$
\begin{aligned}
& \Delta u+g(x, u)=\mu_{0}+\theta \text { in } D \\
& \left.u\right|_{\partial D}=b, \quad \int_{\partial D} \frac{\partial u}{\partial n} d s=0 .
\end{aligned}
$$


Hence, it remains to show that one can choose $\xi_{1}$, so that $\mu=\mu_{0}$ in (2.18). The corresponding $u(x)$ (from (2.18) ) is then a solution of (2.19).

Remark If the domain $D$ is an interval in one dimension, then $c_{0}=\lambda_{2}$. Indeed, suppose $D=(-L, L)$. Represent $u(x) \in W_{0}^{1,2}(-L, L)$ by its Fourier series

$$
u(x)=a_{0}+\Sigma_{n=1}^{\infty} a_{n} \cos \frac{n \pi}{L} x+b_{n} \sin \frac{n \pi}{L} x .
$$

If $\int_{-L}^{L} u(x) d x=0$, then $a_{0}=0$, and hence

$$
\int_{-L}^{L} u^{\prime 2}(x) d x \geq \frac{\pi^{2}}{L^{2}} \int_{-L}^{L} u^{2}(x) d x=0,
$$

i.e., $c_{0}=\frac{\pi^{2}}{L^{2}}$. The Dirichlet eigenvalues are $\lambda_{n}=\frac{n^{2} \pi^{2}}{(2 L)^{2}}$, so that $c_{0}=\lambda_{2}$.

\section{Continuation in $\xi_{1}$}

We have just seen that for each $\xi_{1}$ the problem

$$
\begin{gathered}
\Delta u+g(x, u)=\mu+\theta(x) \text { in } D \\
\left.u\right|_{\partial D}=b, \quad \int_{\partial D} \frac{\partial u}{\partial n} d s=0
\end{gathered}
$$

has a solution $(u, \mu) \in \bar{H}^{2} \times R$, with the average value of $u(x)$ equal to $\xi_{1}$. We now show that all these solutions lie on a unique solution curve, which is globally parameterized by $\xi_{1}$, the average value of the solution $\left(\xi_{1}=\frac{1}{|D|} \int_{D} u(x) d x\right)$.

Theorem 3.1 Assume that the conditions of the Theorem 2.1 hold. Then any solution of (3.1) can be continued in $\xi_{1}$, for all $-\infty<\xi_{1}<\infty$, giving us a curve of solutions $(u, b, \mu)\left(\xi_{1}\right) \in W^{2,2}(D) \times R \times R$. Moreover, for each $\xi_{1}$ there exists a unique solution $(u, b, \mu)$ of (3.1). All solutions of the problem (3.1) lie on a unique continuous solution curve $(u, b, \mu)\left(\xi_{1}\right) \in$ $W^{2,2}(D) \times R \times R$, with $\xi_{1}$ being a global parameter.

Proof: We use the Implicit Function Theorem to show that any solution of (3.1) can be continued in $\xi_{1}$. The proof is essentially the same, as the one above for continuation in $k$. Defining the map

$$
F\left(v, \mu, \xi_{1}\right)=\Delta v+g\left(x, v+\xi_{1}\right)-\mu: \bar{H}^{2} \times R \times R \rightarrow L^{2}(D),
$$

we recast the problem (3.1) in the operator form

$$
F\left(v, \mu, \xi_{1}\right)=\theta(x) .
$$


Similarly to the Theorem 2.1, we apply the Implicit Function Theorem to perform continuation in $\xi_{1}$, by showing that the linearized operator

$$
F_{(v, \mu)}\left(u, \mu, \xi_{1}\right)\left(w, \mu^{*}\right)=\Delta w+g_{u}\left(x, v+\xi_{1}\right) w-\mu^{*}
$$

is one-to-one and onto.

Assume that there are two different pairs of solutions $\left(u_{1}(x), \mu_{1}\right)$ and $\left(u_{2}(x), \mu_{2}\right)$, with $u_{1}(x)$ and $u_{2}(x)$ having the same average value $\xi_{1}^{0}$. These pairs are also solutions of (2.3), with $k=1$. By the Theorem 2.1, we can continue both pairs for decreasing $k$ on two curves of solutions, with fixed average $\xi_{1}^{0}$. These curves do not intersect, since at all points the Implicit Function Theorem applies. At $k=0$, we obtain a contradiction with the uniqueness part of Lemma 2.3 .

\section{Existence of solutions}

We now discuss which values of $\mu$ are covered, as we continue in $\xi_{1} \in$ $(-\infty, \infty)$, which translates into existence results for our problem (1.1).

Theorem 4.1 Assume that the function $g(x, u) \in L^{\infty}(\bar{D} \times R)$ satisfies the conditions (1.4), (2.4) and (2.5). Then the condition (1.5) is necessary and sufficient for the existence of solution for the problem (1.1).

Proof: We proved above that the condition (1.5) is necessary for solvability, we now prove its sufficiency. By the Theorem 2.1, for any $\xi_{1} \in R$, there exists a triple $(u, b, \mu)=(u, b, \mu)\left(\xi_{1}\right)$ solving the problem (1.1), with average of $u(x)$ equal to $\xi_{1}$. As before, we write $u=\xi_{1}+U$, with $U=U\left(\xi_{1}\right)$ satisfying $\int_{D} U d x=0$. By the Theorem 3.1, $\mu=\mu\left(\xi_{1}\right)$ is continuous in $\xi_{1}$, and integrating the equation (1.1), we express

$$
\mu=\frac{1}{|D|} \int_{D} g\left(x, \xi_{1}+U\left(\xi_{1}\right)\right) d x .
$$

We need to show that we can choose $\xi_{1}=\xi_{1}^{0}$, so that $\mu\left(\xi_{1}^{0}\right)=\mu_{0}$. This will follow, once we prove the existence of the limits

$$
\begin{aligned}
\lim _{\xi_{1} \rightarrow \infty} \int_{D} g\left(x, \xi_{1}+U\left(\xi_{1}\right)\right) d x & =\int_{D} g(x, \infty) d x, \\
\lim _{\xi_{1} \rightarrow-\infty} \int_{D} g\left(x, \xi_{1}+U\left(\xi_{1}\right)\right) d x & =\int_{D} g(x,-\infty) d x .
\end{aligned}
$$


In view of (4.1), $U=U\left(\xi_{1}\right)$ is satisfying

$$
\begin{gathered}
\Delta U=-g\left(x, \xi_{1}+U\right)+\frac{1}{|D|} \int_{D} g\left(x, \xi_{1}+U\right) d x+\theta(x), \text { in } D \\
\left.U\right|_{\partial D}=b_{1}, \quad \int_{\partial D} \frac{\partial U}{\partial n} d s=0 \\
\frac{1}{|D|} \int_{D} U(x) d x=0,
\end{gathered}
$$

with $b_{1}=b-\xi_{1}$, a new unknown constant. By Corollary 1 to Lemma 2.1, there is a constant $c$, so that

$$
\left\|U\left(\xi_{1}\right)\right\|_{L^{\infty}(\bar{D})} \leq c, \text { uniformly in } \xi_{1} \in R,
$$

from which the limits (4.2) and (4.3) follow.

The following result is similar to the one in D.G. de Figueiredo and W.-M. Ni [6], who considered the case of Dirichlet problem.

Theorem 4.2 Consider the problem

$$
\begin{gathered}
\Delta u+g(x, u)=\theta(x) \text { in } D \\
\left.u\right|_{\partial D}=b, \quad \int_{\partial D} \frac{\partial u}{\partial n} d s=0,
\end{gathered}
$$

where $\theta(x) \in L^{2}(D)$ is a given function, satisfying $\int_{D} \theta(x) d x=0$. Assume that the function $g(x, u) \in L^{\infty}(\bar{D} \times R)$ satisfies the conditions 2.4 and (2.5), and in addition

$$
u g(x, u)>0, \quad \text { for all } u \in R \text {, and } x \in D .
$$

Then the problem (4.4) has a solution $(u, b) \in W^{2,2}(D) \times R$.

Proof: The proof is similar to the one above. Here we have $\mu^{0}=0$. Using (4.5), we see from (4.1) that $\mu>0(<0)$ for $\xi_{1}$ large and positive (negative). By continuity, we have $\mu\left(\xi_{1}^{0}\right)=0$ for some $\xi_{1}^{0}$.

We also have the following multiplicity result.

Theorem 4.3 Consider the problem

$$
\begin{gathered}
\Delta u+g(x, u)=\mu+\theta(x) \text { in } D \\
\left.u\right|_{\partial D}=b, \quad \int_{\partial D} \frac{\partial u}{\partial n} d s=0,
\end{gathered}
$$

where $\theta(x) \in L^{2}(D)$ is a given function, satisfying $\int_{D} \theta(x) d x=0$. Assume there is a constant $G>0$ such that

$$
u g(x, u)>0, \quad \text { for all }|u|>G, \text { and } x \in D .
$$


Assume that the limits $g(x, \pm \infty)=\lim _{u \rightarrow \pm \infty} g(x, u)$ exist, uniformly in $x \in$ $\bar{D}$, and

$$
g(x, \pm \infty) \equiv 0
$$

Then there exists an interval $\left(\mu_{-}, \mu_{+}\right)$, with $\mu_{-}<0$ and $\mu_{+}>0$, so that for $\mu \in\left(\mu_{-}, \mu_{+}\right) \backslash\{0\}$ the problem (4.6) has at least two solutions $\left(u_{1}, b_{1}\right)$ and $\left(u_{2}, b_{2}\right)$.

Proof: By the Theorem 2.1, for any $\xi_{1} \in R$, there exists a triple $(u, b, \mu)=$ $(u, b, \mu)\left(\xi_{1}\right)$ solving the problem (4.6), with average of $u(x)$ equal to $\xi_{1}$. As in Theorem 4.1, we continue this solution in $\xi_{1}$, paying particular attention to the curve $\mu=\mu\left(\xi_{1}\right)$. From the formula (4.1) and the condition (4.7) it follows that $\mu\left(\xi_{1}\right)$ is positive (negative) for $\left|\xi_{1}\right|$ large and positive (negative). The condition (4.8) implies that $\lim _{\left|\xi_{1}\right| \rightarrow \infty} \mu\left(\xi_{1}\right)=0$, i.e., both sides of the curve $\mu=\mu\left(\xi_{1}\right)$ tend to zero.

\section{References}

[1] A. Ambrosetti and G. Prodi, On the inversion of some differentiable mappings with singularities between Banach spaces, Ann. Mat. Pura Appl. 93 (4), 231-246 (1972).

[2] P. Amster, P.L. De Napoli, and M.C. Mariani, Existence of solutions to $N$-dimensional pendulum-like equations, Electron. J. Differential Equations, No. 125, 8 pp. (electronic) (2004).

[3] H. Berestycki and H. Brezis, On a free boundary problem arising in plasma physics, Nonlinear Anal. 4, no. 3, 415-436 (1980).

[4] A. Castro, Periodic solutions of the forced pendulum equation, Differential equations (Proc. Eighth Fall Conf., Oklahoma State Univ., Stillwater, Okla., 1979), pp. 149-160, Academic Press, New York-LondonToronto, Ont., 1980.

[5] J. Cepicka, P. Drabek and J. Jensikova, On the stability of periodic solutions of the damped pendulum equation, J. Math. Anal. Appl. 209, 712-723 (1997).

[6] D.G. de Figueiredo and W.-M. Ni, Perturbations of second order linear elliptic problems by nonlinearities without Landesman-Lazer condition, Nonlinear Anal. 3 no. 5, 629-634 (1979).

[7] G. Fournier and J. Mawhin, On periodic solutions of forced pendulumlike equations, J. Differential Equations 60, no. 3, 381-395 (1985). 
[8] G. Katriel, Periodic solutions of the forced pendulum: exchange of stability and bifurcations, J. Differential Equations 182, no. 1, 1-50 (2002).

[9] P. Korman, A global solution curve for a class of periodic problems, including the pendulum equation, Z. Angew. Math. Phys, (ZAMP) 58, 749-766 (2007).

[10] P. Korman, Global solution curves for boundary value problems, with linear part at resonance, Nonlinear Anal. 71, no. 7-8, 2456-2467 (2009).

[11] P. Korman, Curves of equiharmonic solutions, and ranges of nonlinear equations, Adv. Differential Equations 14, no. 9-10, 963-984 (2009).

[12] E.M. Landesman and A.C. Lazer, Nonlinear perturbations of linear elliptic boundary value problems at resonance, J. Math. Mech. 19, 609623 (1970).

[13] J. Mawhin, Seventy-five years of global analysis around the forced pendulum equation, In: R. P. Agarwal and F. Neuman and J. Vosmansky (eds.): Proceedings of Equadiff 9, Masaryk University, Brno, pp. 115145 (1997).

[14] R. Ortega, Stability and index of periodic solutions of an equation of Duffing type, Boll. Un. Mat. Ital. B (7), no. 3, 533-546 (1989).

[15] R. Ortega, Topological degree and stability of periodic solutions for certain differential equations, J. London Math. Soc. (2) 42, no. 3, 505516 (1990).

[16] G. Tarantello, On the number of solutions of the forced pendulum equations, J. Differential Equations 80, 79-93 (1989). 